\title{
The Spatial Structure of Macroheterocyclic Compounds, as a Key Factor Affecting the Course of the Macrocyclization Reaction
}

\author{
Konstantin V. Belov, ${ }^{a}$ Ilya E. Eremeev, ${ }^{\text {b }}$ Valentina V. Sobornova, ${ }^{\text {b }}$ \\ Vladimir V. Klochkov, ${ }^{c}$ and Ilya A. Khodova,c@ \\ ${ }^{\mathrm{a}}$ G.A. Krestov Institute of Solution Chemistry of Russian Academy of Sciences, 153045 Ivanovo, Russian Federation \\ ' Ivanovo State University of Chemistry and Technology, 153000 Ivanovo, Russian Federation \\ 'Institute of Physics, Kazan Federal University, 420008 Kazan, Russian Federation \\ ${ }^{\circledR}$ Corresponding authorE-mail: iakh@isc-ras.ru
}

\begin{abstract}
Nowadays the macrocyclization reaction is often used for the synthesis of new macroheterocyclic compounds. This is a one-pot reaction, carried out in one stage, which makes it the most convenient and efficient in the synthesis of complex macroheterocyclic compounds. One of the key parameters in the selection of synthesis conditions is the spatial structure and conformational composition of the synthesized compounds. The fact is that if the certain structure prevails in the solution, then it is the most energetically favorable, i.e. its potential energy is minimal and therefore it is necessary to spend less energy to synthesis of this compound. The structure, obtained during the synthesis process, largely depends on what solvent is used. In this work, it was shown that the conformational composition of the macroheterocyclic model compound in various solvents differs by 10-20\%. This fact must be taken into account when conducting macrocyclization reactions not only for the model compound presented in this paper, but also for other substances consisting of macroheterocyclic molecules, such as crown ethers, cyclosporine, calixarenes, and other classes of compounds having a non-rigid structure.
\end{abstract}

Keywords: Conformers, NMR spectroscopy, the nuclear Overhauser effect (NOE), NOESY, two-dimensional correlation spectroscopy, macroheterocyclic compounds.

\section{Пространственная структура макрогетероциклических соединений, как ключевой фактор, влияющий на протекание реакции макроциклизации}

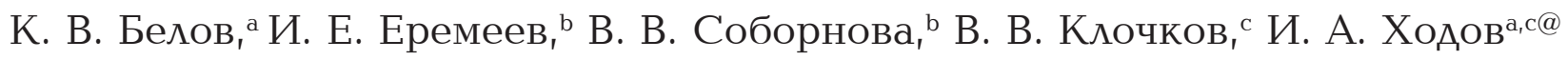 \\ ${ }^{a}$ Институт химии растворов им. Г.А. Крестова Российской академии наук, 153045 Иваново, Россия \\ ${ }^{\mathrm{b}}$ Ивановский государственный химико-технологический университет, 153000 Иваново, Россия \\ ${ }^{\mathrm{c}}$ Казанский (Приволюский) федеральный университет, 420008 Казань, Россия \\ @E-mail: iakh@isc-ras.ru
}

Синтез новыхмакрогетероциклических соединений все чаще основывается на реакциимакрочииклиации. Данный тип реакиий проводится в одну стадию и в одной емкости, что делает её наиболее удобной и эффективной при синтезе сложных макрогетерочиклических соединений. Одним из ключевых параметров при подборе условий синтеза является пространственная структура и конформационный состав синтезируемых соединении. Дело в том, что если определенная структура преобладает в растворе, то она является наиболее энергетически выгодной, т.е. её потенциальная энергия минимальна, и тем самым необходимо затратить меньшее количество энергии на получение данного соединения. Какая структура получится в процессе синтеза во многом зависит от того, какой растворитель будет использован. В данной работе было показано, что конформационный состав макрогетерочииклческого модельного соединения в различных растворителях отличается на 10-20\%. Данный факт необходимо учитывать при проведении реакций макроичилизации не только для модельного соединения, представленного в данной работе, но и для других веществ, состоящих 
из макрогетерочиклических молекул, таких как краун-эфиры, ичиклоспорины, каликсарены и другие классы соединений, имеющих не жесткую структуру.

Ключевые слова: Конформеры, ЯМР спектроскопия, ядерный эффект Оверхаузера, NOESY, двумерная корреляционная спектроскопия, макрогетероциклические соединения.

\section{Введение}

В настоящее время макрогетероциклические соединения играют важную роль в современной органической химии. Существует множество соединений, относящихся к этому классу, например: порфирины, ${ }^{[1-6]}$ корролы, ${ }^{[7-9]}$ фталоцианины, ${ }^{[10-15]}$ краун-эфиры, ${ }^{[16-19]}$ каликсарены, ${ }^{[20-30]}$ циклоспорины, ${ }^{[31-33]}$ порфиразиноиды, ${ }^{[34]}$ олигогетероциклы, ${ }^{[35-37]}$ циклодекстрины, ${ }^{[38-42]}$ порфиразины, ${ }^{[43-45]}$ макролиды, ${ }^{[46-48]}$ циклофаны, ${ }^{[49-52]}$ металлакрауны, ${ }^{[53,54]}$ производные бор-дипиррометенов (BODIPY), ${ }^{[55,56]}$ а также алкалоиды. ${ }^{[57-60]}$ Все соединения, перечисленные выше, а также их производные и комплексы используются в разных областях химической науки и промышленности. В частности, фталоцианины и порфирины могут быть использованы в синтезе красителей, а также являются крайне перспективными объектами для супрамолекулярной и аналитической химии, биомиметики, медицины и фармакологии, ${ }^{[61,62]}$ краун-эфиры могут быть активно задействованы в органическом синтезе и направленной доставке лекарственных препаратов, ${ }^{[19]}$ каликсарены используют для моделирования транспорта воды через клеточную мембрану. ${ }^{[63]}$ Отдельно стоит отметить крупный класс макрогетероциклических соединений - циклоспорины, некоторые из которых используются против отторжения трансплантированных тканей. ${ }^{[32,64]}$ Атропин, морфин, а также ряд других алкалоидов являются важными составляющими современных лекарственных препаратов. ${ }^{[65]}$ Нашли широкое применение и циклодекстрины. Например, некоторые металлоорганические кристаллические каркасы на базе циклодекстринов могут быть использованы для инкапсуляции различных лекарственных препаратов. ${ }^{[66]}$ Также использование циклофанов позволило создавать искусственные ферменты и рецепторы, ${ }^{[49]}$ а металлакрауны используют в качестве одномолекулярных магнитов. ${ }^{[53]}$

Большое количество химических соединений обладает свойством конформационной жесткости. Как правило, такие молекулы являются стабильными и не проявляют конформационную лабильность. В качестве примера конформационно-жестких молекул можно назвать циклодекстрины, фталоцианины или порфирины. Исходя из вышеописанного, всё больший интерес вызывают не конформационно-жесткие молекулы, а конформационно-лабильные за счет широкого спектра использования. Зачастую конформационной лабильностью обладают молекулы макрогетероциклов. Наличие гетероатомов в молекулах гетероциклических соединений обуславливают значительную лабильность их молекул по сравнению с другими органическими соединениями. Это особенно проявляется при наличии нескольких гетероатомов и заместителей в молекуле.
Такие молекулы проявляют наибольшую тенденцию к существованию различных конформационных форм, что обуславливает их специфические свойства.

На сегодняшний день данная тематика является одной из самых востребованных в современной органической химии, но и в тоже время является малоизученной. Поэтому в данной работе мы хотели показать влияние различных растворителей на пространственную структуру конформационно-лабильной молекулы.

\section{Экспериментальная часть}

В работе были исследованы вещества (стрихнин) производства фирмы Sigma-Aldrich 99.99 мас. \%. Для приготовления образцов в качестве растворителей были использованы органические жидкости, в которых атомы водорода замещены атомами дейтерия изотопной чистоты 99+\% фирмы Aldrich: хлороформ- $d_{1} \quad\left(\mathrm{CDCl}_{3}\right)$, диметилсульфоксид- $d_{6}$ (ДМСО) и бензол- $d_{6}\left(\mathrm{C}_{6} \mathrm{D}_{6}\right)$. Все образцы были приготовлены в стандартных 5 мм ампулах без дополнительной очистки вещества.

Все спектры были записаны на многофункциональном импульсном ЯМР спектрометре с Фурье-преобразованием «AVANCE III-500» фирмы «Bruker» (Германия), с рабочей частотой 500 МГц на ядрах ${ }^{1} \mathrm{H}$ и 125 МГц на ядрах ${ }^{13} \mathrm{C}$. Калибровка $90^{\circ}$ импульсов производилась в соответствии с общепринятой методикой поиска 360 импульса, а также при помощи стандартной макросной программы «pulsecal». Применялась цифровая экспоненциальная фильтрация $\mathrm{c} \mathrm{lb}=3-5$ Гц. Отсчет химических сдвигов в спектрах ЯМР ${ }^{1} \mathrm{H}$ и ${ }^{13} \mathrm{C}$ проводился от линий резонанса эталонных жидкостей тетраметилсилана (ТMC), а также от сигналов растворителей (с учетом температуры). Точность определения химических сдвигов составляла \pm 0.01 м.д. Стабилизация магнитного поля осуществлялась по сигналам ядер ${ }^{1} \mathrm{H}$ растворителя. Для решения задачи расшифровки ${ }^{1} \mathrm{H}$ спектров и нахождения ядер, связанных гомоядерным скалярным спин-спиновым взаимодействием, использовалась двумерная ЯМР последовательность TOCSY с последовательностью MLEV-17 в фазочувствительном режиме и импульсным полевым градиентом. Для расшифровки ${ }^{13} \mathrm{C}$ спектров и нахождения, связанных через одну, две и три связи гетероядер ${ }^{1} \mathrm{H}-{ }^{13} \mathrm{C}$ спин-спиновым взаимодействием, использовались двумерные подходы ЯМР (HSQC, НMBC) спектроскопии с последовательностью в фазочувствительном режиме и импульсным полевым градиентом.

Запись одно- и двумерных спектров производилась с использованием рекомендаций, описанных в руководстве по эксплуатации спектрометра. Спектры были оптимизированы на константу спин-спинового взаимодействия HSQC на ${ }^{1} J(\mathrm{C}, \mathrm{H})=145$ Гц, НMBC на ${ }^{\mathrm{n}} J(\mathrm{C}, \mathrm{H})=8$ Гц. Поддержка стабильности температуры производилась с помощью температурной приставки BCU-05 фирмы «Bruker». Показания датчика температуры калибровались перед началом эксперимента в соответствии с инструкцией к прибору. Обработка всех спектров проводилась в рамках программных пакетов 
TopSpin 3.2 фирмы Bruker и ACD/NMR Processor Academic Edition.

Параметры экспериментов в бензоле- $d_{6}$. ${ }^{1} \mathrm{H}$ спектры были получены при помощи стандартной импульсной программы «zg» (TopSpin3.6.1) в спектральном диапазоне 11.06 м.Д. / 5530.97 Гц, число сканирований (NS) - 256, число пустых сканирований (DS) - 2, число точек FID (TD) - 65536, задержка перед подачей радиочастотного электромагнитного импульса (d1) составляла 11.25 секунды, параметр RG (Receiver gain, усиление приемника) - 114, время эксперимента 48 минут.

${ }^{13} \mathrm{C}$ спектры были получены при помощи стандартной импульсной программы «zgpg30» (TopSpin3.6.1) в спектральном диапазоне 236.62 м.д. / 29761.90 Гц, число сканирований (NS) - 8192, число пустых сканирований (DS) - 4, число точек FID (TD) - 16384, задержка перед подачей радиочастотного электромагнитного импульса (d1) составляла 2 секунды, параметр RG (Receiver gain, усиление приемника) - 161, время эксперимента 5 часов 19 минут.

${ }^{1} \mathrm{H}-{ }^{13} \mathrm{C}$ HSQC спектры были получены при помощи стандартной импульсной программы «hsqcetgpsi2» (TopSpin3.6.1) в спектральном диапазоне, соответствующем одномерным спектрам ${ }^{1} \mathrm{H}$ и ${ }^{13} \mathrm{C}$, число сканирований (NS) - 8, число пустых сканирований (DS) - 16, число точек FID (TD) 1024(F2)/256(F1), задержка перед подачей радиочастотного электромагнитного импульса (d1) составляла 7 секунд, параметр RG (Receiver gain, усиление приемника) - 2050, время эксперимента 4 часа 4 минуты.

${ }^{1} \mathrm{H}-{ }^{1} \mathrm{H}$ TOCSY спектры были получены при помощи стандартной импульсной программы «mlevgpphprzf» (TopSpin3.6.1) в спектральном диапазоне, соответствующем одномерному спектру ${ }^{1} \mathrm{H}$, число сканирований (NS) - 8, число пустых сканирований (DS) - 16, число точек FID (TD) 2048(F2)/256(F1), задержка перед подачей радиочастотного электромагнитного импульса (d1) составляла 11.25 секунды, параметр RG (Receiver gain, усиление приемника) - 114, были зарегистрированы 3 спектра TOCSY с различными временами смешивания $(0.02,0.06$ и 0.1 сек), среднее время каждого эксперимента 6 часов 30 минут.

${ }^{1} \mathrm{H}-{ }^{1} \mathrm{H}$ NOESY спектры были получены при помощи стандартной импульсной программы «noesygpphzs» (TopSpin3.6.1) в спектральном диапазоне, соответствующем одномерному спектру ${ }^{1} \mathrm{H}$, число сканирований (NS) - 20, число пустых сканирований (DS) - 16, число точек FID (TD) - 2048(F2)/256(F1), задержка перед подачей радиочастотного электромагнитного импульса (d1) составляла 11.25 секунды, параметр RG (Receiver gain, усиление приемника) - 114, были зарегистрированы 8 спектров NOESY с различными временами смешивания $(0.05,0.1,0.15,0.2,0.25,0.3,0.35$ и 0.4 сек), среднее время каждого эксперимента 16 часов.

Параметры экспериментов в хлороформе-д1. ${ }^{1} \mathrm{H}$ спектры были получены при помощи стандартной импульсной программы «zg» (TopSpin3.6.1) в спектральном диапазоне 10.74 м.д. / 5376.34 Гц, число сканирований (NS) - 400, число пустых сканирований (DS) - 0, число точек FID (TD) - 65536, задержка перед подачей радиочастотного электромагнитного импульса (d1) составляла 4 секунды, параметр RG (Receiver gain, усиление приемника) - 36, время эксперимента 28 минут.

${ }^{13} \mathrm{C}$ спектры были получены при помощи стандартной импульсной программы «zgpg30» (TopSpin3.6.1) в спектральном диапазоне 198.76 м.д. / 25000.00 Гц, число сканирований (NS) - 2048, число пустых сканирований (DS) - 0, число точек FID (TD) - 65536, задержка перед подачей радиочастотного электромагнитного импульса (d1) составляла 3 секунды, параметр RG (Receiver gain, усиление приемника) - 161, время эксперимента 2 часа 29 минут.

${ }^{1} \mathrm{H}^{13} \mathrm{C}$ HSQC спектры были получены при помощи стандартной импульсной программы «hsqcetgpsi2» (TopSpin3.6.1) в спектральном диапазоне, соответствующем одномерным спектрам ${ }^{1} \mathrm{H}$ и ${ }^{13} \mathrm{C}$, число сканирований (NS) - 128, число пустых сканирований (DS) - 16, число точек FID (TD) 1024(F2)/256(F1), задержка перед подачей радиочастотного электромагнитного импульса (d1) составляла 1.65 секунды, параметр RG (Receiver gain, усиление приемника) - 161, время эксперимента 16 часов 2 минуты.

${ }^{1} \mathrm{H}-{ }^{1} \mathrm{H}$ TOCSY спектры были получены при помощи стандартной импульсной программы «mlevphpr.2» (TopSpin3.6.1) в спектральном диапазоне, соответствующем одномерному спектру ${ }^{1} \mathrm{H}$, число сканирований (NS) - 16, число пустых сканирований (DS) - 16, число точек FID (TD) - 2048(F2)/512(F1), задержка перед подачей радиочастотного электромагнитного импульса (d1) составляла 1.65 секунды, параметр RG (Receiver gain, усиление приемника) - 36, были зарегистрированы 3 спектра TOCSY с различными временами смешивания (0.02, 0.06 и 0.1 сек), среднее время каждого эксперимента 4 часа 30 минут.

${ }^{1} \mathrm{H}-{ }^{1} \mathrm{H}$ NOESY спектры были получены при помощи стандартной импульсной программы «noesygpphzs» (TopSpin3.6.1) в спектральном диапазоне, соответствующем одномерному спектру ${ }^{1} \mathrm{H}$, число сканирований (NS) - 32, число пустых сканирований (DS) - 16, число точек FID (TD) - 2048(F2)/256(F1), задержка перед подачей радиочастотного электромагнитного импульса (d1) составляла 4 секунды, параметр RG (Receiver gain, усиление получателя) - 36, были зарегистрированы 8 спектров NOESY с различными временами смешивания $(0.05,0.1,0.15,0.2,0.25,0.3,0.35$ и 0.4 сек), среднее время каждого эксперимента 10 часов.

Параметры экспериментов в диметилсульфоксиде- $d_{6}$. ${ }^{1} \mathrm{H}$ спектры были получены при помощи стандартной импульсной программы «zg» (TopSpin3.6.1) в спектральном диапазоне 14.00 м.д. / 7002.80 Гц, число сканирований (NS) - 64, число пустых сканирований (DS) - 2, число точек FID (TD) - 65536, задержка перед подачей радиочастотного электромагнитного импульса (d1) составляла 6.5 секунды, параметр RG (Receiver gain, усиление приемника) - 128, время эксперимента 10 минут.

${ }^{13} \mathrm{C}$ спектры были получены при помощи стандартной импульсной программы «zgpg30» (TopSpin3.6.1) в спектральном диапазоне 236.62 м.д. / 29761.90 Гц, число сканирований (NS) - 2048, число пустых сканирований (DS) - 4, число точек FID (TD) - 16384, задержка перед подачей радиочастотного электромагнитного импульса (d1) составляла 2 секунды, параметр RG (Receiver gain, усиление приемника) - 287, время эксперимента 13 часов 18 минут.

${ }^{1} \mathrm{H}-{ }^{13} \mathrm{C}$ HSQC спектры были получены при помощи стандартной импульсной программы «hsqcetgpsi2» (TopSpin3.6.1) в спектральном диапазоне, соответствующем одномерным спектрам ${ }^{1} \mathrm{H}$ и ${ }^{13} \mathrm{C}$, число сканирований (NS) - 8, число пустых сканирований (DS) - 16, число точек FID (TD) 1024(F2)/256(F1), задержка перед подачей радиочастотного электромагнитного импульса (d1) составляла 7 секунд, параметр RG (Receiver gain, усиление приемника) - 2050, время эксперимента 4 часа 4 минуты.

${ }^{1} \mathrm{H}-{ }^{1} \mathrm{H}$ TOCSY спектры были получены при помощи стандартной импульсной программы «mlevgpphprzf» (TopSpin3.6.1) в спектральном диапазоне, соответствующем одномерному спектру ' $\mathrm{H}$, число сканирований (NS) - 16, число пустых сканирований (DS) - 16, число точек FID (TD) 2048(F2)/256(F1), задержка перед подачей радиочастотного электромагнитного импульса (d1) составляла 6.5 секунды, параметр RG (Receiver gain, усиление получателя) - 203, были зарегистрированы 3 спектра TOCSY с различными временами смешивания (0.02, 0.06 и 0.1 сек), среднее время каждого эксперимента 7 часов 30 минут.

${ }^{1} \mathrm{H}-{ }^{1} \mathrm{H}$ NOESY спектры были получены при помощи стандартной импульсной программы «noesygpphzs» 
(TopSpin3.6.1) в спектральном диапазоне, соответствующем одномерному спектру ${ }^{1} \mathrm{H}$, число сканирований (NS) - 32, число пустых сканирований (DS) - 16, число точек FID (TD) - 2048(F2)/256(F1), задержка перед подачей радиочастотного электромагнитного импульса (d1) составляла 6.5 секунды, параметр RG (Receiver gain, усиление приемника) - 203, были зарегистрированы 8 спектров NOESY с различными временами смешивания $(0.05,0.1,0.15,0.2,0.25,0.3,0.35$ и 0.4 сек), среднее время каждого эксперимента 15 часов.

\section{Результаты и обсуждение}

Известно, что макрогетероциклы достаточно непростые соединения, обладающие не только сложным строением, но и наличием специфических свойств. Зачастую в таких молекулах можно наблюдать наличие ионной, водородной и металлической связей, также большую роль играют многоцентровые связи и наличие мостиковых типов взаимодействий. Исходя из этого, макрогетероциклы представляют большой интерес в сфере искусственного синтеза. ${ }^{[67]}$ Из-за их сложного строения необходимо правильно подобрать методику и все необходимые условия для успешного проведения синтеза, а также учитывать такие параметры, как конформации макроциклов с лабильной структурой.

В данной работе мы продемонстрировали метод по определению пространственной структуры и конформационных распределений сложных макрогетероциклических соединений при подборе параметров синтеза на примере молекулы стрихнина (Рисунок 1). Стоит отметить, что стрихнин является модельным соединением, вместо которого можно использовать любой макрогетероцикл.

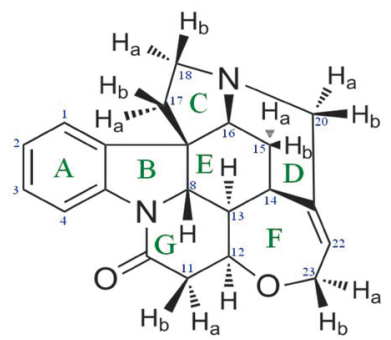

Рисунок 1. Молекулярная структура стрихнина.

Долгое время структура данного соединения считалась жесткой, однако, согласно данным исследований последних лет было установлено, что молекула стрихнина склонна к конформационной подвижности. ${ }^{[68]}$ За последние годы были проведены исследования на основе рентгеновской дифракции, остаточного диполь-дипольного взаимодействия RDC и других методов, подтверждающих и уточняющих строение и конформационные формы молекулы стрихнина. ${ }^{[69]}$

Одним из эффективных методов для решения подобного рода задач является спектроскопия ядерного магнитного резонанса (ЯМР), а в частности подход ядерного эффекта Оверхаузера, который лежит в основе эксперимента NOESY.
Данный метод очень чувствителен, что является как очевидным преимуществом, так и в некотором роде недостатком, так как малейшие изменения в расчетных или экспериментальных параметрах (скоростей кроссрелаксации, интегральных интенсивностей сигналов и т.д.) приводят к серьезным погрешностям в определении конформационного распределения.

Однако, при должной математической обработке и вычислении расчетных величин, подход с использованием NOESY легко применим и информативен для расчета конформационных популяций, даже таких сложных систем, как стрихнин. Метод NOESY позволяет наблюдать взаимодействия водородов по типу ${ }^{1} \mathrm{H}-{ }^{1} \mathrm{H}$, пространственно близко расположенных. Спектр NOESY дает представление о пространственных корреляциях, обусловленных спин-решеточной релаксацией.

Определение экспериментальных расстояний в спектрах NOESY основано на корреляции скоростей кросс-релаксации $\sigma_{\mathrm{ij}}$ и расстояний между ядерными спинами $r_{\text {ij }}$ Данная зависимость может быть определена как: $\sigma_{\mathrm{ij}}=1 / r_{\mathrm{ij}}{ }^{6}$. Тогда как экспериментальное межъядерное расстояние описывается следующей формулой:

$$
r_{i j}=r_{0}\left(\frac{\sigma_{0}}{\sigma_{i j}}\right)^{\frac{1}{6}},
$$

где $r_{0}$ и $\sigma_{0}$ относятся к референсному расстоянию ${ }^{1} \mathrm{H}-{ }^{1} \mathrm{H}$, не подверженному смене конформаций. Обычно референсное расстояние $r_{0}$ определяется из квантово-химических расчетов структуры. Однако данный метод дает недостаточно точную информацию о межъядерных расстояниях. Существует более сложный подход, основанный на интегральных интенсивностях кросс-пиков в спектрах NOESY, нормированных по интенсивностям диагональных пиков..$^{[23,70,71]}$ Интенсивность сигналов в спектрах NOESY связана с величиной $\tau_{\mathrm{m}}$ (временем смешивания), что позволяет построить линейную зависимость $I\left(\tau_{\mathrm{m}}\right)$ :

$$
\bar{I}\left(\tau_{m}\right)=\frac{1}{2}\left(\frac{1}{n_{j}}\left|\frac{a_{i j}\left(\tau_{m}\right)}{a_{i i}\left(\tau_{m}\right)}\right|+\frac{1}{n_{i}}\left|\frac{a_{j i}\left(\tau_{m}\right)}{a_{j j}\left(\tau_{m}\right)}\right|\right),
$$

где $n_{\mathrm{j}}, n_{\mathrm{i}}-$ параметры, указывающие на количество протонов, входящих в состав определяемой группы, $a_{\mathrm{ij}}, a_{\mathrm{ji}}-$ параметры, определяющие интенсивность кросс-пиков в спектрах 2D NOESY, $a_{\mathrm{ii}}, a_{\mathrm{jj}}$ - параметры, определяющие интенсивность диагональных сигналов в спектрах 2D NOESY.

Применение данной математической модели позволяет частично устранить влияние дрейфа экспериментального параметра.

Из приведенной выше зависимости (2) возможно определить скорость кросс-релаксации для пары взаимодействующих протонов (Рисунок 2).

Также следует учитывать анизотропное вращение молекулы. При взаимодействии двух эквивалентных спинов экспериментально определяемое расстояние является усредненным для всех существующих форм молекулы в растворе. 


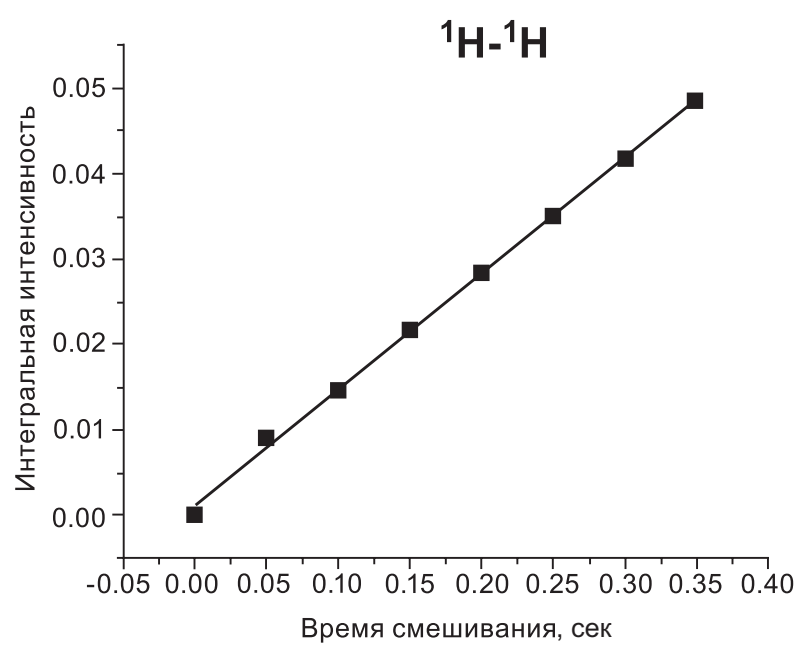

Рисунок 2. Зависимость интегральной интенсивности от времени смешивания.

Таким образом, для расчета процентного распределения конформеров в растворе необходимо иметь представление о скоростях кросс-релаксации для пар протонов $\sigma_{\mathrm{ij}}$ и $\sigma_{0}$, конформационно-зависимые и референсные расстояния, полученные из квантовохимических расчетов $r_{0}$ и $r_{1}$, а также конформационнозависимое расстояние, полученное из эксперимента $r_{\mathrm{ij}}$ :

$$
X_{1}=\frac{r_{1}^{6}\left(r_{i j}{ }^{6}-r_{0}{ }^{6}\right)}{r_{i j}{ }^{6}\left(r_{1}^{6}-r_{0}{ }^{6}\right)},
$$

где $X_{1}$ - процентная доля одного из конформеров. ${ }^{[72-75]}$

Представленный подход уже был апробирован ранее для ряда сложных гетероциклических соединений и позволил получить информацию не только на качественном, но и на количественном уровне. ${ }^{[76-78]}$ Таким образом, использование метода NOESY для установления конформационной подвижности позволит получить новую информацию для характеристики особенностей преднуклеационного состояния биологически активных соединений.

В 2010 году Craig P. Butts и соавторы ${ }^{[79]}$ проводят исследование характеристик структуры молекулы стрихнина на основе эксперимента NOE в различных растворителях. Полученные экспериментальные данные несколько расходятся с величинами, полученными из расчетов. Данный факт авторы связывают с вероятной конформационной подвижностью молекулы, а различия в экспериментальных расстояниях при переходе от растворителя к растворителю - с влиянием вязкости. Затем, в этом же году в работе C. Butts и соавторов на основе все того же подхода NOE доказывается существование второй минорной конформации молекулы в растворе хлороформа- $d_{1}$ и бензола- $d_{6}$ с популяцией $2.5 \% .^{[80]}$ Данное исследование подтверждает способность NOE эксперимента различать незначительные вклады в динамические структурные ансамбли. В общей сложности было измерено 55 межпротонных расстояний, однако, для некоторых расстояний, таких как H11b-H23b, ошибка в определении составляла $14.9 \%$, что в значительной мере отличается от средней ошибки по всему набору данных. Данное различие расчетных (DFT) и экспериментальных (NOE) данных авторы связывают с существованием второй конформационной формы, в которой данное расстояние меньше, и этот второй конформер вносит значительный вклад в интенсивность NOE.

В работе M. Schmidt и соавторов ${ }^{[81]}$ предложен иной способ определения количественного содержания конформеров в растворе. Авторы считают, что метод NOE не является надежным для решения подобной задачи. Молекула стрихнина при условиях, предложенных в работе Craig P. Butts, находится в быстром обмене и, следовательно, интенсивности NOE усреднены и зависят от скорости химического обмена. В работе проведен подбор параметров для наиболее точной оптимизации геометрии в рамках DFT анализа. В рамках эксперимента был проведен анализ конформационной гибкости молекулы стрихнина на основе ЯМР эксперимента с варьированием температуры, чтобы нивелировать влияние процессов химического обмена. В ходе работы было определено процентное распределение конформеров в растворе хлороформа- $d_{1}$, где популяция второго конформера составляет $5.7 \%$.

Данные работы свидетельствуют о существовании конформационной формы молекулы стрихнина, где центр конформационной активности приходится на кольцо F в структуре.

Для удобства расчетов примем конформеры, описанные в данных работах как эквивалентные и обозначим как F (Рисунок 3A). Однако существуют и другие гипотезы относительно конформационной подвижности данного объекта.

B работе G. Bifulco и соавторов ${ }^{[82]}$ идет речь о других возможных конформационных формах с центром конформационной активности в кольце С. Авторы используют метод структурного анализа на основе $J$-связи (JBCA). Данный метод основан на двугранной угловой зависимости констант связи, включая геминальные (связанные с одним атомом) и вицинальные (связанные с соседними атомами) ( $\left.{ }^{3} J_{\mathrm{HH}},{ }^{3} J_{\mathrm{CH}},{ }^{2} J_{\mathrm{CH}}\right)$. Использование метода ЈВСА в сочетании с ЯМР подходом позволило авторам обнаружить второй менее стабильный конформер с популяцией всего $0.11 \%$ при $25^{\circ} \mathrm{C}$.

Одной из последних работ по данному вопросу является статья G. Tomba и соавторов. ${ }^{[83]}$ Моделирование, проведенное в данной работе, позволило получить профили свободной энергии, на основе которых были выявлены три торсионных угла с различными значениями. В работе описана стратегия применения метода остаточных диполярных связей (RDC) для определения конформационной подвижности некоторых молекул. Авторы позиционируют данный метод, как наиболее точный и универсальный способ определения количественного (процентного) содержания конформеров. Таким образом, авторами было выдвинуто предположение о существование трех различных конформационных форм со следующими популяциями в растворе хлороформа $(94.9 \%, 4.9 \%$ и $0.2 \%$, соответственно). 


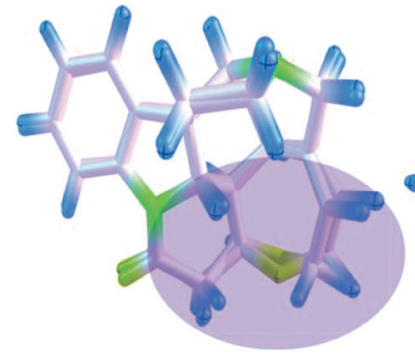

A

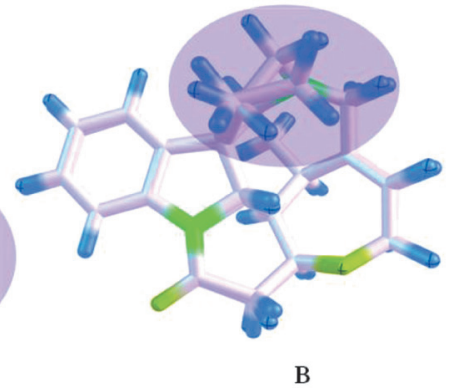

B
Рисунок 3. Молекулярные структуры конформеров стрихнина из работ C. Butts и M. Schmidt (слева, группа F), G. Bifulco и G. Tomba (справа, группа C). Области указывают на центры конформационной подвижности.

Данные конформеры отнесем к группе С (Рисунок 3В).

В обзорной работе A. Kolmer и соавторов ${ }^{[69]}$ проведен подробный анализ предыдущих работ. Авторы проводят поиск возможных ошибок в усреднении данных и предлагают провести анализ на основе более новых данных RDC и эксперимента NOE с целью уточнения и более подробного исследования конформационной лабильности молекулы стрихнина. В частности, одной из предстоящих целей является детальное изучение гибкости кольца $\mathrm{C}$, описанное в работе G. Bifulco.

Исходя из анализа литературных данных, можно сделать вывод, что на сегодняшний день известно пять конформационных форм молекулы стрихнина в растворе, однако, причины проявления подобной подвижности молекулы в растворе до сих пор не установлены. В данной работе все конформационные формы были разделены на 2 группы (F и C) в соответствии с центром конформационной активности (кольцо $\mathrm{F}$ и кольцо $\mathrm{C}$ ). K каждой группе принадлежит по 2 конформера (F1/F2 и $\mathrm{C} 1 / \mathrm{C} 2)$ в соответствии с литературными данными. Конформер «С3» из работы ${ }^{[83]}$ не учитывался ввиду малой популяции.

Далее представлены результаты анализа конформационных распределений молекулы стрихнина в трех различно-полярных растворителях (бензол- $d_{6}$, хлороформ- $d_{1}$, ДМСO- $d_{6}$ ) методом 2D NOESY с различными временами смешивания и сравнение с литературными данными.

Таким образом, на первом этапе были получены одно- $\left({ }^{1} \mathrm{H},{ }^{13} \mathrm{C}\right)$ (см. SI.1-SI.7) и двумерные $\left({ }^{1} \mathrm{H}-{ }^{13} \mathrm{C}\right.$ HSQC, ${ }^{1} \mathrm{H}-{ }^{1} \mathrm{H}$ TOCSY, ${ }^{1} \mathrm{H}-{ }^{1} \mathrm{H}$ NOESY) спектры стрихнина (см. SI.8-SI.18). Одномерные протонные спектры можно условно разделить на три области: к первой области относятся сигналы, лежащие в диапазоне 6.5-9.0 м.д., принадлежащие протонам бензольного кольца в структуре молекулы, сигнал Н22 принадлежит второй области спектра, и 17 сигналов - третья область (0-5 м.д.). В связи с тем, что в углеродных спектрах нет явного визуального разделения на группы, описание углеродного спектра можно ограничить двумя компонентами, включающими в себя по 9 и 12 сигналов, соответствующих атомам углерода.
На следующем этапе были выявлены конформационно-зависимые и референсные расстояния в структуре молекулы. Для данных расстояний были рассчитаны скорости кросс-релаксации на основе корреляции интегральной интенсивности соответствующего кросс-пика и времени смешивания (см. SI.19-SI.30). Исходя из попыток подбора референсных и конформационно-зависимых расстояний, можно сделать предположение, что данные расстояния должны быть связаны, и желательно, чтобы они находились в одной плоскости.

Для конформеров группы «F» при расчете распределений в бензоле в качестве конформационнозависимого расстояния была выбрана пара протонов H14-H13 $\left(r_{1}=2.41190 \AA, r_{2}=2.51746 \AA\right)\left(r_{1}=2.41099 \AA\right.$, $\left.r_{2}=2.51635 \AA\right)$, скорость кросс-релаксации для которой $\sigma=0.03315$. В качестве калибровочного (референсного) расстояния - Н14-Н15a $\left(r_{1}=2.54967 \AA, r_{2}=2.55810\right.$ $\AA)\left(r_{1}=2.54900 \AA, r_{2}=2.55370 \AA\right)$, скорость кроссрелаксации для которого $\sigma=0.02389$. Таким образом, для конформеров группы F были получены следующие конформационные распределения (F1/F2 = 97.1 \% / $2.9 \% ; \mathrm{F} 1 / \mathrm{F} 2=96.1 \% / 3.9 \%$ ), исходя из расчетов C. Butts и M. Schmidt, соответственно.

Для конформеров группы «С» при расчете распределений в бензоле в качестве конформационно-зависимого расстояния была выбрана пара протонов Н14-Н13 $\left(r_{1}=2.41193 \AA, r_{2}=2.47436 \AA\right)$ из расчетов G. Bifulco и расстояние Н11a-H13 $\left(r_{1}=3.56319 \AA, r_{2}=3.00343 \AA\right)$ из расчетов G. Tomba, скорость кросс-релаксации для которых составляет $\sigma=0.03315$ и $\sigma=0.00368$, соответственно. В качестве калибровочных расстояний были выбраны H14-H15a $\left(r_{1}=2.54967 \AA, r_{2}=2.57228\right.$ $\AA)$ и H11a-H11b $\left(r_{1}=1.69189 \AA, r_{2}=1.72667 \AA\right)$, скорость кросс-релаксации для которых $\sigma=0.02389$ и $\sigma=0.12722$, соответственно (Рисунок 4). Таким образом, для конформеров группы С были получены следующие конформационные распределения (C1/C2 = 79.3 \% / 20.7 \%; $\mathrm{C} 1 / \mathrm{C} 2=76.5 \%$ / $23.5 \%$ ), исходя из расчетов G. Bifulco и G. Tomba, соответственно.

Для конформеров группы «F» при расчете распределений в хлороформе в качестве конформационнозависимого расстояния была выбрана пара протонов H8-H11b $\left(r_{1}=2.67203 \AA, r_{2}=2.30382 \AA\right)\left(r_{1}=2.65464 \AA\right.$, $r_{2}=2.26598 \AA$ ), скорость кросс-релаксации для которой $\sigma=0.03000$. В качестве калибровочного расстояния H8-H13 $\left(r_{1}=3.00765 \AA, r_{2}=3.02990 \AA\right)\left(r_{1}=3.00959 \AA\right.$, $\left.r_{2}=3.03385 \AA\right)$, скорость кросс-релаксации для которого $\sigma=0.01234$. Таким образом, для конформеров группы F были получены следующие конформационные распределения $(\mathrm{F} 1 / \mathrm{F} 2=88.2 \% / 11.8$ \%; F1/F2 = $92.6 \%$ / $7.4 \%$ ), исходя из расчетов C. Butts и M. Schmidt, соответственно.

Для конформеров группы «С» при расчете распределений в хлороформе в качестве конформационнозависимого расстояния была выбрана пара протонов H8-H11b $\left(r_{1}=2.67113 \AA, r_{2}=2.89917 \AA\right)\left(r_{1}=2.45725 \AA\right.$, $r_{2}=3.34247 \AA$ ), скорость кросс-релаксации для которой составляет $\sigma=0.03000$. В качестве калибровочных расстояний были выбраны H11b-H13 $\left(r_{1}=3.66229\right.$ $\AA, r_{2}=3.68452 \AA$ ) из расчетов G. Bifulco и Н8-H13 


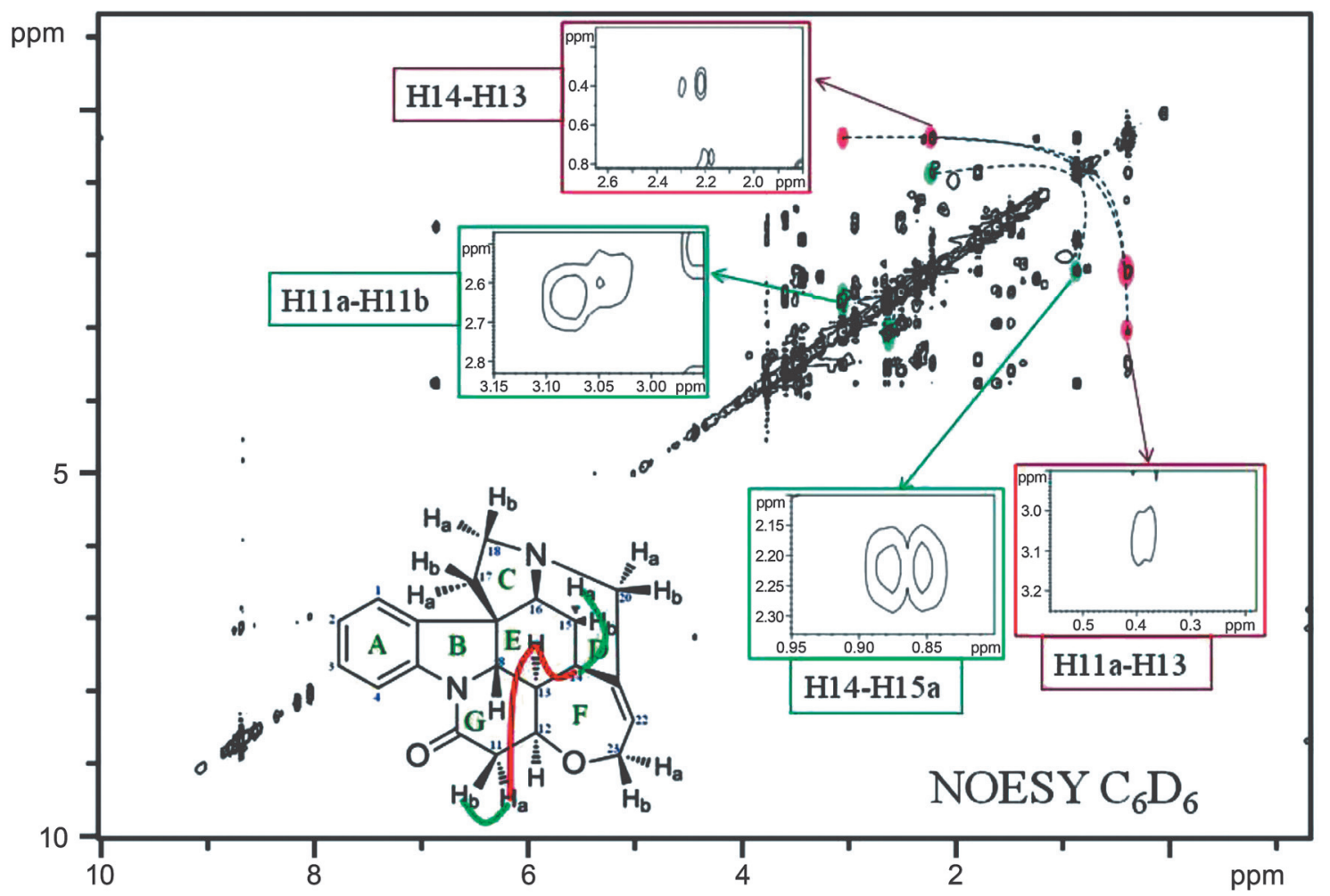

Рисунок 4. Спектр ${ }^{1} \mathrm{H}-{ }^{1} \mathrm{H}$ NOESY молекулы стрихнина в бензоле- $d_{6}$.

$\left(r_{1}=2.92460 \AA, r_{2}=2.93416 \AA\right)$ из расчетов G. Tomba, скорость кросс-релаксации для которых $\sigma=0.00463$ и $\sigma=0.01234$, соответственно (Рисунок 5). Таким образом, для конформеров группы «С» были получены следующие конформационные распределения (C1/ $\mathrm{C} 2=89.2 \% / 10.8 \%$; $\mathrm{C} 1 / \mathrm{C} 2=81.8 \% / 18.2 \%$ ), исходя из расчетов G. Bifulco и G. Tomba, соответственно.

Для конформеров группы «F» при расчете распределений в диметилсульфоксиде в качестве конформационно-зависимого расстояния была выбрана пара протонов Н12-H14 $\left(r_{1}=2.73364 \AA, r_{2}=2.97123 \AA\right)\left(r_{1}=2.71862 \AA\right.$, $\left.r_{2}=2.96235 \AA\right)$, скорость кросс-релаксации для которой $\sigma=0.02929$. В качестве калибровочного расстояния H14-H15b $\left(r_{1}=2.48434 \AA, r_{2}=2.49907 \AA\right)\left(r_{1}=2.47997 \AA\right.$, $r_{2}=2.49344 \AA$ ), скорость кросс-релаксации для которого $\sigma=0.06052$. Таким образом, для конформеров группы F были получены следующие конформационные распределения $(\mathrm{F} 1 / \mathrm{F} 2=88.1 \% / 11.9 \%$; F1/F2 = 83.5\% / $16.5 \%$ ), исходя из расчетов C. Butts и M. Schmidt, соответственно.

Для конформеров группы «С» при расчете распределений в диметилсульфоксиде в качестве конформационно-зависимого расстояния была выбрана пара протонов Н20a-H15b $\left(r_{1}=3.71368 \AA, r_{2}=2.41231\right.$ $\AA$ ) из расчетов G. Bifulco и расстояние Н12-H13 $\left(r_{1}=2.25340 \AA, r_{2}=2.51748 \AA\right)$ из расчетов G. Tomba, скорость кросс-релаксации для которых составляет $\sigma=0.14229$ и $\sigma=0.07981$, соответственно. В качестве калибровочных расстояний были выбраны H14-H15b $\left(r_{1}=2.48434 \AA, r_{2}=2.47990 \AA\right)$ и Н14-Н13 $\left(r_{1}=2.36937 \AA\right.$, $\left.r_{2}=2.41580 \AA\right)$, скорость кросс-релаксации для которых $\sigma=0.06052$ и $\sigma=0.010105$, соответственно (Рисунок 6).
Таким образом, для конформеров группы С были получены следующие конформационные распределения $(\mathrm{C} 1 / \mathrm{C} 2=69.4 \% / 30.6 \%$; C1/C2 = $76.4 \%$ / $23.6 \%)$, исходя из расчетов G. Bifulco и G. Tomba, соответственно.

Проведем сравнение полученных нами данных и результатов, найденных в соответствующих литературных источниках (Таблица 1). Так, для конформеров группы «F», рассматриваемых в работах C. Butts и M. Schmidt, полученные популяции в бензоле- $d_{6}$ (97.1\%/2.9\% $\quad$ (Butts), $\quad 96.1 \% / 3.9 \% \quad$ (Schmidt)) и хлороформе- $d_{1}(88.2 \% / 11.8 \%$ (Butts), $92.6 \% / 7.4 \%$ (Schmidt)) коррелируют с данными, полученными в этих работах $(97.5 \% / 2.5 \%$ (Butts)), (94.3\%/5.7\% (Schmidt)). Однако для конформеров группы «С» корреляция не настолько явная: конформер С1 является преобладающим, но его содержание несколько ниже, чем содержание, приведенное в литературе. Данный факт может быть обусловлен тем, что в работе ${ }^{[83]}$ используется три конформера молекулы, в то время как в данной работе были выбраны лишь два. Вклад третьего минорного конформера влияет на распределение, приведенное в данной работе.

Кроме того, наблюдается перераспределение популяций в зависимости от используемого растворителя. Так, например, в ряду бензол- $d_{6}$, хлороформ- $d_{1}$, ДМСО- $d_{6}$ наблюдаются следующие распределения конформеров: F1 (97.1 \%, 88.2 \%, 88.1\%), F2 (2.9\%, $11.8 \%$, $11.9 \%)$, C1 (79.0 \%, $89.2 \%, 69.4 \%)$, C2 (20.7\%, $10.8 \%$, $30.6 \%$ по расчетам C. Butts и G. Bifulco; F1 (96.1\%, $92.6 \%, 83.5 \%)$, F2 (3.9\%, $7.4 \%, 16.5 \%)$, C1 (76.5\%, $81.8 \%, 76.4 \%)$, C2 (23.5\%, $18.2 \%, 23.6 \%)$ по расчетам M. Schmidt и G. Tomba. 


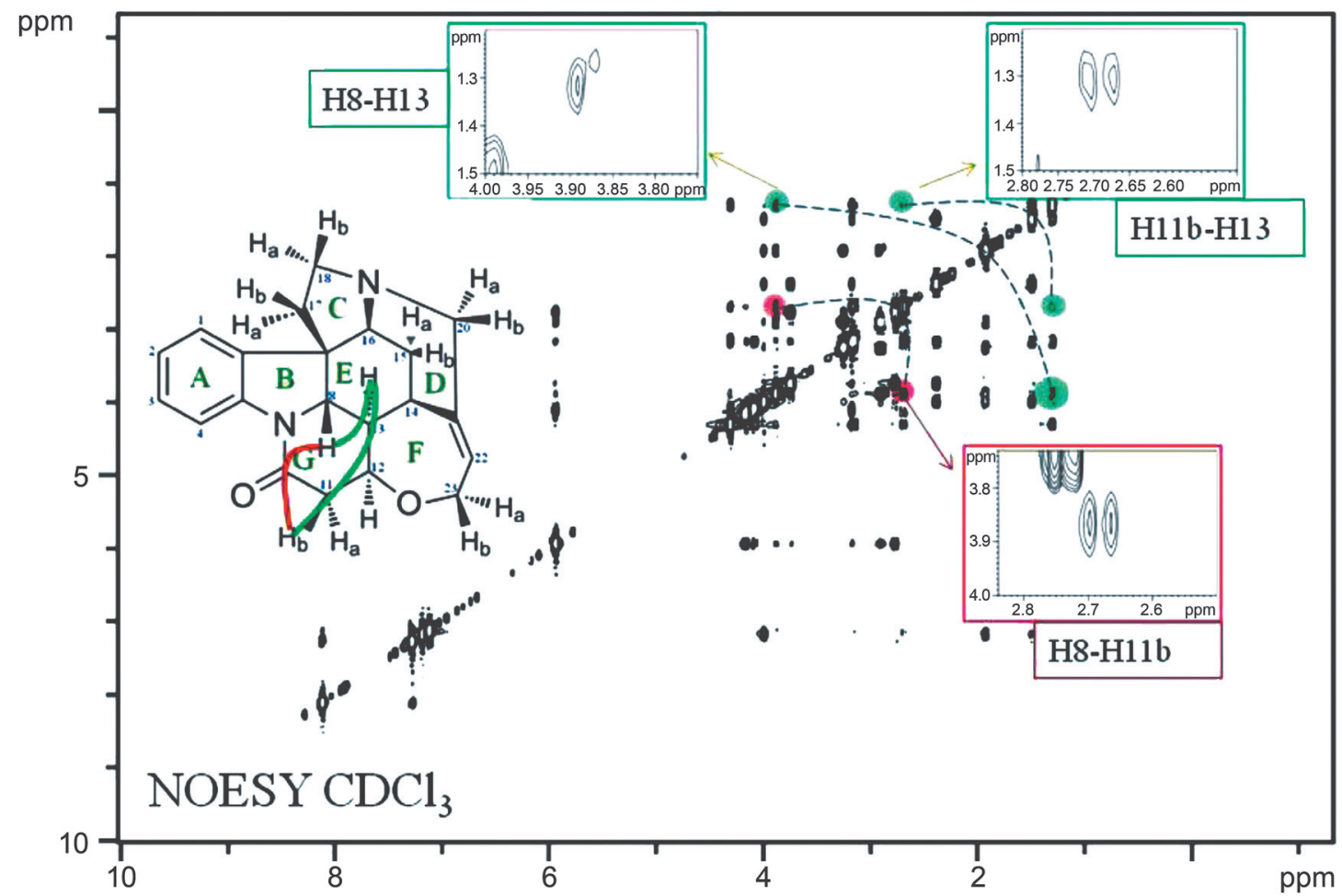

Рисунок 5. Спектр ${ }^{1} \mathrm{H}-{ }^{1} \mathrm{H}$ NOESY молекулы стрихнина в хлороформе- $d_{1}$.

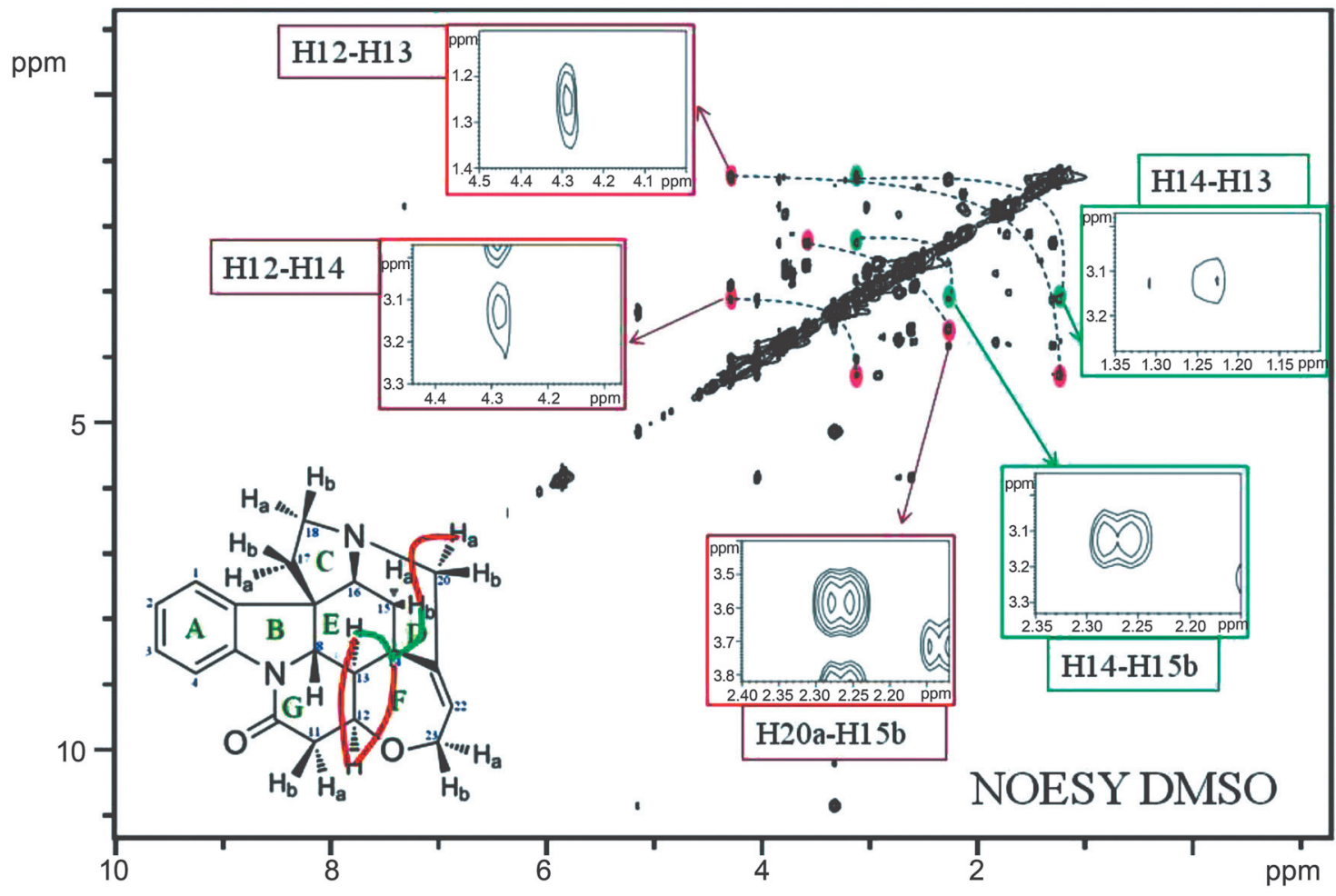

Рисунок 6. Спектр ${ }^{1} \mathrm{H}-{ }^{1} \mathrm{H}$ NOESY молекулы стрихнина в диметилсульфоксиде- $d_{6}$.

Данный метод в перспективе будет полезен тем, кто занимается органическим синтезом, а в частности реакциями макроциклизации. На сегодняшний день существует множество подходов к синтезу макрогетероциклических соединений, одним из самых перспективных является макроциклизация. ${ }^{[84]}$ В органической химии насчитывается большое количество таких реакций, и отличаются они лишь подбором условий проведения синтеза и растворителем. Но стоит отметить то, что такие реакции, как правило, протекают в одной колбе. 
Таблица 1. Результаты расчетов конформационных распределений молекулы стрихнина в различных растворителях.

Bifulco/Tomba
Растворители

Например, метод Келлога является достаточно распространённым способом макроциклизации краун-эфиров. ${ }^{[85]}$ Основа данного метода заключается в использовании в качестве основания карбоната цезия, за счет которого получают, как правило, неплохие выходы синтезируемого продукта. Кроме того, существует метод получения макроциклов, основанный на внутримолекулярном олефинировании альдегида енолятом кислоты по Хорнеру-Эммонсу в модификации Стилле и приводящий к смеси изомеров. Данный метод применяется в синтезе макролидов и его структурных аналогов. ${ }^{[86]}$ Также стоит упомянуть реакцию аннелирования по Робинсону, ${ }^{[8]}$ в которой после протекания первой стадии с донором и акцептором Михаэля, полученный продукт сразу же подвергается циклизации и образует женский половой гормон - эстрон. Один из других способов получения макроциклов - это введение азотсодержащего фрагмента в уже готовую молекулу макроциклического антибиотика лейкомицина с помощью различных иминонитрозо-производных по реакции Дильса-Альдера. ${ }^{[88,89]}$ Дополнительным примером эффективного получения макроциклов является замыкание кольца макролида с помощью протекающей по механизму внутримолекулярной реакции между нуклеофильным малонат-анионом и хлораллильным фрагментом ациклического предшественника.

Особое место среди реакций макроциклизации занимает реакция, предложенная Мицунобу ${ }^{[00]}$ (Рисунок 7). В классическом варианте реакция Мицунобу является эстерификацией спиртов нуклеофилом в присутствии диалкилазодикарбоксилата (DEAD) и трифенилфосфина.

В макроциклизации Мицунобу реакция проходит по похожему механизму, что и в классическом вари-

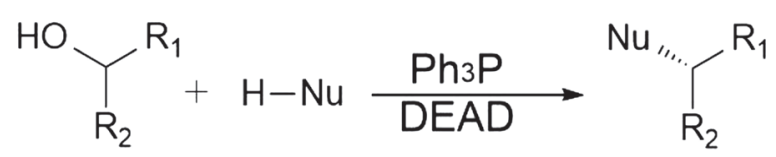

Рисунок 7. Схема протекания реакции Мицунобу.

анте, но образуя сложный циклический фрагмент. ${ }^{[90,91]}$ Данный метод зачастую применим в синтезе макрогетероциклов и славится за счет упрощения синтеза сложных циклических фрагментов молекулы. Так, долгий и многостадийный синтез изохинолинов по методу Померанца-Фрича представляет собой прямой метод получения ароматических производных изохинолина в условиях реакции Мицунобу. В работе ${ }^{[92]}$ авторам удалось синтезировать молекулу стрихнина при помощи метода макроциклизации Мицунобу, ${ }^{[90]}$ тем самым упростив подход к синтезу данного соединения за счет построения перегруженной центральной кольцевой системы за один этап.

Так как макроциклизация Мицунобу имеет общие характеристики с другими реакциями макроциклизации, то логично предположить, что выбор растворителя и структура играют также большую роль в проведении синтеза другими методами макроциклирования. В настоящей работе было проведено исследование молекулы стрихнина в трех растворителях. Известно, что для одного и того же соединения в различных растворителях могут наблюдаться кардинальные различия в распределении конформеров, что в итоге может влиять как на свойства получаемого продукта, так и на процент выхода в ходе реакции. Дело в том, что реакция куда охотнее проходит с низкоэнергетическим конформером, нежели с менее устойчивым. Поэтому различия 
структуры необходимо учитывать при подборе условий синтеза методом макроциклизации.

\section{Заключение}

В данной работе нами был предложен метод исследования особенностей пространственной структуры макрогетероциклических соединений, который позволяет точно установить не только пространственную структуру молекулы в растворе, но и конформерный состав исследуемой молекулы. Было показано, что состав и процентное содержание конформеров напрямую зависят от выбранного растворителя. Различие между населенностями конформеров может составлять от 10 до 20 \% в зависимости от выбранного растворителя. Также в случае конформеров группы «С» наблюдались расхождения полученных в эксперименте данных с теоретическими. Это связано с тем, что метод является достаточно чувствительным и позволяет регистрировать малейшие изменения в конформационном составе исследуемого соединения, что, безусловно, является плюсом данной методики. В общем случае, данный метод дает бесценную информацию о структуре, что будет полезно при выборе оптимального пути синтеза для получения необходимого конформера в различных растворителях.

Благодарности. This work was supported by the Russian Foundation for Basic Research (grants No. 18-29-06008 and 18-03-00255); by Ministry of Education and Science of the Russian Federation (contract No. 01201260481 and 0120095082) and by grant of Council on grants of the President of the Russian Federation (project MK-1409.2019.3) in part of NMR methodology, and by the Russian Science Foundation (SE., project No. 18-73-10088) in part of NOESY experiment. The work was sponsored by a subsidy under the auspices of state support to KFU for improving its competitiveness among leading global science and education centers. The NMR spectroscopy experiment was performed using the molecular fluid spectroscopy facility (http://www.ckp-rf.ru/ usu/503933/) of G.A. Krestov Institute of Solution Chemistry of the Russian Academy of Sciences (ISC RAS) (Russia).

\section{Список литературы}

\section{References}

1. Mamardashvili G.M., Kaigorodova E.Y., Khodov I.A., Scheblykin I., Mamardashvili N.Z., Koifman O.I. J. Mol. Liq. 2019, 293, 111471.

2. Mamardashvili G.M., Maltceva O.V., Lazovskiy D.A., Khodov I.A., Borovkov V., Mamardashvili N.Z., Koifman O.I. J. Mol. Liq. 2019, 277, 1047-1053.

3. Khodov I.A., Maltceva O.V., Klochkov V.V., Koifman O.I., Mamardashvili N.Z. New J. Chem. 2017, 41, 7932-7937.

4. Khodov I.A., Nikiforov M.Y., Alper G.A., Mamardashvili G.M., Mamardashvili N.Z., Koifman O.I. J. Mol. Struct. 2015, 1081, 426-430.

5. Bichan N.G., Tyulyaeva E.Y., Khodov I.A., Lomova T.N. J. Mol. Struct. 2014, 1061, 82-89.
6. Zhabanov Y.A., Tverdova N.V., Giricheva N.I., Girichev G.V., Stuzhin P.A. J. Porphyrins Phthalocyanines 2017, 21, 439-452.

7. Mondal S., Garai A., Naik P.K., Adha J.K., Kar S. Inorg. Chim. Acta 2020, 501, 119300.

8. Nardis S., Mandoj F., Stefanelli M., Paolesse R. Coord. Chem. Rev. 2019, 388, 360-405.

9. Mondal S., Naik P.K., Adha J.K., Kar S. Coord. Chem. Rev. 2019, 400, 213043.

10. Wöhrle D., Schnurpfeil G., Makarov S.G., Kazarin A., Suvorova O.N. Macroheterocycles 2012, 5, 191-202.

11. Urbani M., De La Torre G., Nazeeruddin M.K., Torres T. Chem. Soc. Rev. 2019, 48, 2738-2766.

12. Aggarwal A., Samaroo D., Jovanovic I.R., Singh S., Tuz M.P., Mackiewicz M.R. J. Porphyrins Phthalocyanines 2019, 23, 729-765.

13. Cooper S.A.L., Graepel K.W., Steffens R.C., Dennis D.G., Cambroneo G.A., Wiggins R.Q., Ruppel J.V., Snyder N.L. J. Porphyrins Phthalocyanines 2019, 23, 850-855.

14. Roguin L.P., Chiarante N., García Vior M.C., Marino J. Int. J. Biochem. Cell Biol. 2019, 114, 105575.

15. Silaghi-Dumitrescu R., Makarov S.V.,Uta M.-M., Dereven'kov I.A., Stuzhin P.A. New J. Chem. 2011, 35, 1140-1145.

16. Ren X., Zhang J., Cheng M., Wang Q., Jiang J., Wang L. Tetrahedron Lett. 2018, 59, 2197-2204.

17. Yu X.-H., Cai X.-J., Hong X.-Q., Tam K.Y., Zhang K., Chen W.-H. Future Med. Chem. 2019, 11, 1091-1106.

18. Móczár I., Huszthy P. Chirality 2019, 31, 97-109.

19. Chehardoli G., Bahmani A. Supramol. Chem. 2019, 31, 221-238.

20. Luo J., Zheng Y. Prog. Chem. 2018, 30, 601-615.

21. Español E.S., Villamil M.M. Biomolecules 2019, 9(3), 90.

22. García-Rio L., Basílio N. Curr. Opin. Colloid Interface Sci. 2019, 44, 225-237.

23. Gadiev T.A., Khairutdinov B.I., Antipin I.S., Klochkov V.V. Appl. Magn. Reson. 2006, 30, 165-173.

24. Stoikov I.I., Ibragimova D.S., Antipin I.S., Konovalov A.I., Gadiev T.A., Khairutdinov B.I., Karataeva F.K., Klochkov V.V. Russ. Chem. Bull. 2004, 53, 2269-2275.

25. Karataeva F.K., Rezepova M.V., Zhukov A.Y., Stoikov I.I., Antipin I.S., Klochkov V.V. Russ. J. Gen. Chem. 2009, 79, 466-474.

26. Stoikov I.I., Yantemirova A.A., Nosov R.V., Julmetov A.R., Klochkov V.V., Antipin I.S., Konovalov A.I. Mendeleev Commun. 2011, 21, 41-43.

27. Stoikov I.I., Yantemirova A.A., Nosov R.V., Rizvanov I.K., Julmetov A.R., Klochkov V.V., Antipin I.S., Konovalov A.I., Zharov I. Org. Biomol. Chem. 2011, 9, 3225-3234.

28. Karataeva F.K., Vagizova R.R., Stoikov I.I., Antipin I.S., Klochkov V.V. Russ. J. Gen. Chem. 2013, 83, 698-702.

29. Gadiev T.A., Khairutdinov B.I., Shaikhutdinov R.A., Karatayeva F.K., Aganov A.V., Klochkov V.V. Appl. Magn. Reson. 2003, 25, 347-352.

30. Klochkov V.V., Khairutdinov B.I., Klochkov A.V., Tagirov M.S., Thiele C.M., Berger S., Vershinina I.S., Stoikov I.I., Antipin I.S., Konovalov A.I. Russ. Chem. Bull. 2004, 53, 1466-1470.

31. Efimov S.V., Klochkov V.V. Magn. Reson. Solids 2012, 14, 12202.

32. Efimov S.V., Karataeva F.K., Aganov A.V., Berger S., Klochkov V.V. J. Mol. Struct. 2013, 1036, 298-304.

33. Efimov S., Zgadzay Y., Klochkov V. Appl. Magn. Reson. 2014, 45, 1225-1235.

34. Kozlov A.V., Stuzhin P.A. Macroheterocycles 2014, 7, 170-173.

35. Baeuerle P., Mitschke U., Mena-Osteritz E., Sokolowski M., Mueller D., Gross M., Meerholz K. In: Proc. SPIE - Int. Soc. Opt. Eng. 1998. pp. 32-39. 
36. Luo F.-T., Bajji A.C. J. Chin. Chem. Soc. 2000, 47, 257-261.

37. Mitschke U., Debaerdemaeker T., Bäuerle P. Eur. J. Org. Chem. 2000, 2000, 425-437.

38. Haimhoffer A., Rusznyák Á., Réti-Nagy K., Vasvári G., Váradi J., Vecsernyés M., Bácskay I., Fehér P., Ujhelyi Z., Fenyvesi F. Sci. Pharm. 2019, 87(4), 33.

39. Zhang Y.-M., Liu Y.-H., Liu Y. Adv. Mater. 2020, 32(14), 1907226.

40. Kim D.-H., Lee S.-E., Pyo Y.-C., Tran P., Park J.-S. J. Pharm. Investig. 2020, 50, 17-27.

41. Tian B., Hua S., Liu J. Carbohydr. Polym. 2020, 232, 115805.

42. Usacheva T., Kabirov D., Beregova D., Gamov G., Sharnin V., Biondi M., Mayol L., D'Aria F., Giancola C. J. Therm. Anal. Calorim. 2019, 138, 417-424.

43. Ivanova S.S., Lebedeva I.A., Somov N.V., Marova A.A., Popov A., Stuzhin P.A. Macroheterocycles 2019, 12, 276-281.

44. Lermontova S.A., Grigoryev I.S., Peskova N.N., Ladilina E.Y., Lyubova T.S., Plekhanov V.I., Grishin I.D., Balalaeva I.V., Klapshina L.G. Macroheterocycles 2019, 12, 268-275.

45. Kosov A.D., Dubinina T.V., Seliverstov M.Y., Tomilova L.G., Zefirov N.S. Macroheterocycles 2016, 9, 201-205.

46. Zhang Y., Dai J., Jian H., Lin J. Microbiol. Immunol. 2019, 63, 343-349.

47. Lees K.A., Orlandi R.R., Oakley G., Alt J.A. Immunol. Allergy Clin. North Am. 2020, 40, 303-315.

48. Pincheira M.A., Bacharier L.B., Castro-Rodriguez J.A. Pediatr. Drugs 2020, 22, 217-228.

49. Gol'dfarb Y.I., Belen'kii L.I. Russ. Chem. Rev. 1960, 29, 214-235.

50. David O.R.P. Tetrahedron 2012, 68, 8977-8993.

51. Ghasemabadi P.G., Yao T., Bodwell G.J. Chem. Soc. Rev. 2015, 44, 6494-6518.

52. Spenst P., Würthner F. J. Photochem. Photobiol. C Photochem. Rev. 2017, 31, 114-138.

53. Zaleski C.M., Depperman E.C., Kampf J.W., Kirk M.L., Pecoraro V.L. Angew. Chem. Int. Ed. 2004, 43, 3912-3914.

54. Zabrodina G.S., Katkova M.A., Baranov E.V., Zhigulin G.Y., Ketkov S.Y. Macroheterocycles 2019, 12, 300-306.

55. Ksenofontov A.A., Guseva G.B., Antina E.V., Khodov I.A., Vyugin A.I. Sens. Actuators, B Chem. 2017, 251, 858-868.

56. Ksenofontov A.A., Bichan N.G., Khodov I.A., Antina E.V., Berezin M.B., Vyugin A.I. J. Mol. Liq. 2018, 269, 327-334.

57. McNaught A., Wilkinson A. IUPAC. Compend. Chem. Terminol. 2nd edn. (the "Gold Book") 2014, 1307, 2014.

58. Roblot F., Hocquemiller R., Cave A. Bull. Soc. Chim. Fr. 1984, NO. 3-4II.

59. Buron F., Plé N., Turck A., Queguiner G. J. Org. Chem. 2005, 70, 2616-2621.

60. Nailya S., Zhanat S., Roza Z., Raushan T. J. Int. Pharm. Res. 2019, 46, 694-696.

61. Zhang G.-D., Harada A., Nishiyama N., Jiang D.-L., Koyama H., Aida T., Kataoka K. J. Controlled Release 2003, 93, 141-150.

62. Beletskaya I., Tyurin V.S., Tsivadze A.Y., Guilard R., Stern C. Chem. Rev. 2009, 109, 1659-1713.

63. Thallapally P.K., Lloyd G.O., Atwood J.L., Barbour L.J. Angew. Chem. Int. Ed. 2005, 44, 3848-3851.

64. Borel J.F., Di Padova F., Mason J., Quesniaux V., Ryffel B., Wenger R. Pharmacol. Rev. 1989, 41, 239-242.
65. Shamsa F., Esfahani H.R., Gamooshi R.A. Tehran Univ. Med. J. 2008, 66, 237-241.

66. Kritskiy I., Volkova T., Surov A., Terekhova I. Carbohydr. Polym. 2019, 216, 224-230.

67. Chuchuryukin A.V., Chase P.A., Dijkstra H.P., Suijkerbuijk B.M.J.M., Mills A.M., Spek A.L., Van Klink G.P.M., Van Koten G. Adv. Synth. Catal. 2005, 347, 447-462.

68. Thiele C. M. J. Org. Chem. 2004, 69, 7403-7413.

69. Kolmer A., Edwards L.J., Kuprov I., Thiele C.M. J. Magn. Reson. 2015, 261, 101-109.

70. Williamson M.P., Neuhaus D. J. Magn. Reson. 1987, 72, 369-375.

71. Fejzo J., Zolnai Z., Macura S., Markley J.L. J. Magn. Reson. 1989, 82, 518-528.

72. Khodov I.A., Nikiforov M.Y., Alper G.A., Blokhin D.S., Efimov S.V., Klochkov V V., Georgi N. J. Mol. Struct. 2013, $1035,358-362$.

73. Khodov I.A., Efimov S.V., Klochkov V.V., Alper G.A., Batista De Carvalho L.A.E. Eur. J. Pharm. Sci. 2014, 65, 65-73.

74. Khodov I.A., Belov K.V., Efimov S.V., De Carvalho L.A.E.B. AIP Conf. Proc. 2019, 2063, 040007.

75. Khodov I.A., Kiselev M.G., Efimov S.V., Klochkov V.V. J. Magn. Reson. 2016, 266, 67-68.

76. Gamov G.A., Khodov I.A., Belov K.V., Zavalishin M.N., Kiselev A.N., Usacheva T.R., Sharnin V.A. J. Mol. Liq. 2019, 283, 825-833.

77. Nikitina L.E., Pavelyev R.S., Startseva V.A., Kiselev S.V., Galiullina L.F., Aganova O.V., Timerova A.F., Boichuk S.V., Azizova Z.R., Klochkov V.V., et al. J. Mol. Liq. 2020, 301, 112366.

78. Khodov I., Dyshin A., Efimov S., Ivlev D., Kiselev M. J. Mol. Liq. 2020, 113113.

79. Butts C.P., Jones C.R., Towers E.C., Flynn J.L., Appleby L., Barron N. J. Org. Biomol. Chem. 2011, 9, 177-184.

80. Butts C.P., Jones C.R., Harvey J.N. Chem. Commun. 2011, 47, 1193-1195.

81. Schmidt M., Reinscheid F., Sun H., Abromeit H., Scriba G.K.E., Sönnichsen F.D., John M., Reinscheid U.M. Eur. J. Org. Chem. 2014, 2014, 1147-1150.

82. Bifulco G., Riccio R., Martin G.E., Buevich A.V, Williamson R.T. Org. Lett. 2013, 15, 654-657.

83. Tomba G., Camilloni C., Vendruscolo M. Methods 2018, 148, 4-8.

84. Martí-Centelles V., Pandey M.D., Burguete M.I., Luis S.V., Chem. Rev. 2015, 115, 8736-8834.

85. Rogers R.D., Henry R.F., Ochrymowycz L.A., Toske S.G. $J$. Incl. Phenom. Mol. Recognit. Chem. 1993, 15, 145-152.

86. Ishmuratov G.Yu., Yakovleva M.P., Mingaleeva G.R., Tolstikov A.G. Macroheterocycles 2011, 4, 270-310.

87. Yarlagadda S., Sankaram G.S., Balasubramanian S., Subba Reddy B.V. Org. Lett. 2018, 20, 4195-4199.

88. Zhang C., Jiao H., Jia W. Comput. Theor. Chem. 2020, 1175, 112734.

89. Dyan O.T., Borodkin G.I., Zaikin P.A. Eur. J. Org. Chem. 2019, 2019, 7271-7306.

90. Mitsunobu O. Synth. 1981, 1981, 1-28.

91. Swamy K.C.K., Kumar N.N.B., Balaraman E., Kumar K.V.P.P. Chem. Rev. 2009, 109, 2551-2651.

92. Cannon J.S., Overman L.E. Angew. Chemie, Int. Ed. 2012, 51, $4288-4311$. 